UCRL-TR-217980

LCLS-TN-06-1

January 11, 2006

\title{
The Physics Analysis of a Gas Attenuator with Argon as a Working Gas ${ }^{1}$
}

\author{
D.D. Ryutov, R.M. Bionta, M.A. McKernan, S. Shen, and J.W. Trent \\ Lawrence Livermore National Laboratory, Livermore, CA, 94551
}

\begin{abstract}
A gas attenuator is an important element of the LCLS facility. The attenuator must operate in a broad range of x-ray energies, provide attenuation coefficient between 1 and $10^{4}$ with the accuracy of $1 \%$ and, at the same time, be reliable and allow for many months of un-interrupted operation. S. Shen [1] has recently carried out a detailed design study of the attenuator based on the use of nitrogen as a working gas. In this note we assess the features of the attenuator based on the use of argon. We concentrate on the physics issues, not the design features.
\end{abstract}

\section{Disclaimer}

This document was prepared as an account of work sponsored by an agency of the United States Government. Neither the United States Government nor the University of California nor any of their employees, makes any warranty, express or implied, or assumes any legal liability or responsibility for the accuracy, completeness, or usefulness of any information, apparatus, product, or process disclosed, or represents that its use would not infringe privately owned rights. Reference herein to any specific commercial product, process, or service by trade name, trademark, manufacturer, or otherwise, does not necessarily constitute or imply its endorsement, recommendation, or favoring by the United States Government or the University of California. The views and opinions of authors expressed herein do not necessarily state or reflect those of the United States Government or the University of California, and shall not be used for advertising or product endorsement purposes.

\footnotetext{
1 This work was performed under the auspices of the U.S. Department of Energy by University of California, Lawrence Livermore National Laboratory under Contract W-7405-Eng-48.

Work supported in part by the DOE Contract DE-AC02-76SF00515. This work was performed in support of the LCLS project at SLAC.
} 


\section{The physics analysis of a gas attenuator with argon as a working gas}

\section{Introduction}

A gas attenuator is an important element of the LCLS facility. The attenuator has to operate in a broad range of $\mathrm{x}$-ray energies, provide attenuation coefficient between 1 and $10^{4}$ with the accuracy of $1 \%$ and, at the same time, be reliable and allow for many months of un-interrupted operation. A detailed design study of the attenuator based on the use of nitrogen as a working gas has been recently carried out by S. Shen [1].

In this note we assess the features of the attenuator based on the use of argon. We concentrate on the physics issues; the design features will probably be not that different from the aforementioned nitrogen attenuator. Although specific results obtained in our note pertain to argon, the general framework (and many equations obtained) are applicable also to the nitrogen attenuator.

In the past, an analysis of the attenuator based on the use of a noble gas has already been carried out [2]. This analysis was performed for an extremely stringent set of specifications. In particular, a very large diameter for the unobstructed x-ray beam was set $(1 \mathrm{~cm})$ to accommodate the spontaneous radiation; the attenuator was supposed to cover the whole range of energies of the coherent radiation, from $800 \mathrm{eV}$ to $8000 \mathrm{eV}$; the maximum attenuation was set at the level of $10^{4}$; the use of solid attenuators was not allowed, as well as the use of rotating shutters. The need to reach a sufficient absorption at the high-energy end of the spectrum predetermined the choice of Xe as the working gas (in order to have a reasonable absorption at a not-too-high pressure). A sophisticated differential pumping system that included a Penning-type ion pump was suggested in order to minimize the gas leak into the undulator/accelerator part of the facility. A high cost of xenon meant also that an efficient (and expensive) gas-recovery system would have to be installed.

The main parameter that determined the high cost and the complexity of the system was a large radius of the orifice. The present viewpoint allows for much smaller size of the orifice, $a=1.5 \mathrm{~mm}$.

The use of solid attenuators is also allowed for a higher-energy end of the spectrum. It is, therefore, worthwhile to reconsider various parameters of the gas attenuator for these much less stringent conditions. As a working gas we consider now the argon, which, on the one hand, provides reasonable absorption lengths and, on the other hand, is inexpensive enough to be exhausted into the atmosphere (no need for recovery).

We concentrate on the processes in the main attenuation cell and just outside it, not touching upon a performance of the differential pumping system. The graphs presented in this report can serve for a general orientation only, not for getting exact numerical values of various parameters. 


\section{The absorption properties of Argon}

The absorption properties of argon [3] are illustrated by Fig.1 where the attenuation factor $A$ is shown for various beam energies. The other relevant parameters for argon are presented in Table 1.

Table 1. Some parameters of argon

\begin{tabular}{|c|c|c|c|c|c|}
\hline $\begin{array}{c}\text { Atomic } \\
\text { number }\end{array}$ & $\begin{array}{c}\text { Atomic } \\
\text { radius }\end{array}$ & $\begin{array}{c}\text { Collision } \\
\text { cross- section }\end{array}$ & $\begin{array}{c}\text { Density at } \\
\text { normal } \\
\text { cond. }\end{array}$ & $\begin{array}{c}\text { Sound speed } \\
\text { at normal } \\
\text { cond. }\end{array}$ & $\begin{array}{c}\text { Viscosity, } \\
\text { at } 0^{0} \mathrm{C}[4]\end{array}$ \\
\hline $\mathrm{A}=40$ & $r_{a}=0.98 \AA$ & $\begin{array}{c}\sigma=1.21 \cdot 10^{-15} \\
\mathrm{~cm}^{2}\end{array}$ & $\begin{array}{c}\rho_{0}=1.78 \cdot \\
10^{-3} \mathrm{~g} / \mathrm{cm}^{3}\end{array}$ & $s_{0}=320 \mathrm{~m} / \mathrm{s}$ & $\begin{array}{c}\eta=2.1 \cdot 10^{-4} \\
\mathrm{~g} / \mathrm{s} \cdot \mathrm{cm}\end{array}$ \\
\hline
\end{tabular}

Figure 2 shows the line density of argon required to reach an attenuation of $10^{4}$. Assuming that the length of the main attenuation cell is $L=6 \mathrm{~m}$, one sees that, to reach the highest attenuation of $A=10^{4}$, one has to have the pressures in this cell in the range between $\sim 2$ torr and $\sim 60$ torr.

\section{Evaluation of the gas throughput}

The mean free path for the argon atoms, evaluated as

$$
\lambda=\frac{1}{n \sigma},
$$

where $n$ is the particle density, and $\sigma$ is the collision cross-section, see Table 1 . In the pressure range 0.1 torr $<p<100$ torr, $\lambda$ lies between a few hundred microns and a fraction of a micron (Fig. 3). This means that the gas flow is collisional in the sense that the mean free path is much shorter than the orifice radius $a$. This, in turn, means that the gas exhaust through each of the orifices will occur in a hydrodynamic manner (not in a Knudsen manner); the discharge through each of the orifices can then be evaluated by Eq. (14) of Ref. [2]:

$$
Q=\pi a^{2} s_{0} n_{0}\left(\frac{2}{\gamma+1}\right)^{\frac{\gamma+1}{2(\gamma-1)}},
$$

where the quantities bearing a subscript " 0 " refer to the attenuation cell, and $\gamma$ is the adiabatic index ( $\gamma=5 / 3$ for argon). For further reference, we present also the local sound speed (the flow velocity) at the sonic transition point,

$$
s^{*}=s_{0} \sqrt{\frac{2}{\gamma+1}} \approx 0.87 s_{0}
$$

and the gas density at this point,

$$
n^{*}=n_{0}\left(\frac{2}{\gamma+1}\right)^{\frac{1}{\gamma-1}} \approx 0.65 n_{0}
$$

(see, e.g., [5] for detailed derivation). The sound speed $s_{0}$ at normal conditions is presented in Table1. 
Taking $a$ from Eq. (1) and assuming that the argon in the cell is at a room temperature, one can rewrite Eq. (3) as:

$$
Q_{0}(\text { atoms } / s)=4.6 \cdot 10^{19} p_{0}(\text { torr }),
$$

where $p_{0}$ is the pressure in the central cell. Alternatively, this can be presented as:

$Q_{0}(g /$ hour $)=11 p_{0}($ torr $)$.

\section{Cost of Argon}

The right scale in Fig. 2 shows the gas consumption for $L=6 \mathrm{~m}$ and the attenuation $A=10^{4}$. At the highest energies, the consumption may reach $\sim 1 \mathrm{~kg} /$ hour $(1 \mathrm{~kg}$ is equivalent to approximately 20 cubic feet at normal conditions). This seems to be acceptable in terms of the operation cost. Indeed, the cost of 100 cubic feet of argon is in the range of \$2. This would mean that, even under the most challenging conditions (8 $\mathrm{keV} \mathrm{X}$ rays, $10^{4}$ attenuation coefficient), the cost of argon for 1 day of the LCLS operation will not exceed $\$ 10$.

\section{A more detailed characterization of the gas flow}

Thus far, we have been concerned only with the amount of gas spent. We now consider in more detail the structure of the gas flow. We assume that the gas inlets are situated near the mid-plane of the attenuation cell and are distributed symmetrically with respect to this plane (or, alternatively, there is just one inlet situated exactly in the midplane), Fig. 4. The average (over the cross-section) axial velocity in the mid-plane is obviously zero. It becomes essentially constant (vs. z) in the zone between the inlets and the very close proximity of the exit hole. The flow streamlines are shown in Fig. 4.

The average axial velocity in the intermediate zone (which occupies the main part of the attenuation cell) is, obviously

$$
\mathrm{v}=\frac{Q}{\pi b^{2} n_{0}}=s_{0}\left(\frac{a}{b}\right)^{2}\left(\frac{2}{\gamma+1}\right)^{\frac{\gamma+1}{2(\gamma-1)}} \approx 0.56 s_{0}\left(\frac{a}{b}\right)^{2}
$$

where $Q$ is given by Eq. (3), and $b$ is the tube radius (Fig. 4). For the tube radius $b=8 \mathrm{~cm}$, one has

$$
\mathrm{v}=2 \times 10^{-4} \mathrm{~s}_{0} \approx 6.4 \mathrm{~cm} / \mathrm{s} \text {. }
$$

(9)

In other words, the flow is deeply subsonic and, therefore, essentially incompressible. By applying the Bernoulli equation, one can show that the density variation is $\delta n / n_{0} \sim v^{2} / s_{0}{ }^{2}<$ $2 \times 10^{-6}$. This, in turn, means, that, when evaluating the absorption coefficient, one can assume that the gas density is uniform over the main part of the attenuation cell. This is especially important at the highest attenuation coefficients $\left(A=10^{4}\right)$, see Sec. 8 .

The flow pattern in the vicinity of the exit orifice is shown in Fig. 5. On the inner side this is (approximately) a radial converging flow. We will be interested in the on-axis density variation. For the radially-converging flow, one can readily obtain the following approximate density distribution [5]:

$$
n \approx n_{0}\left(1-\frac{\gamma-1}{\gamma+1} \frac{a^{2}}{z^{2}+a^{2}}\right)^{\frac{1}{\gamma-1}} \approx n_{0}\left(\frac{z^{2}+0.75 a^{2}}{z^{2}+a^{2}}\right)^{3 / 2},
$$


where $z$ is the distance along the axis from the end-plate.

In the outer space, we have a divergent supersonic jet, with the tangent of the divergence angle determined by the ratio of the local sound speed to the flow velocity. This ratio is close to 1 . So, in this zone the density decreases with $z$ as, roughly,

$$
n=n * \frac{a^{2}}{z^{2}+a^{2}}=n_{0} \frac{0.65 a^{2}}{z^{2}+a^{2}} .
$$

We will use Eqs. (10) and (11) later, when evaluating the contribution of the transition region to the attenuation coefficient.

Now we evaluate the Reynolds number of the flow in the main part of the attenuator and in the nozzle. The Reynolds number for the pipe flow can be defined as

$$
\mathrm{Re}=\frac{\rho r \mathrm{v}}{\eta}
$$

where $\rho$ is the mass density of the gas. The viscosity $\eta$ is independent of the density and weakly depends on the temperature. So we will approximate it just by the constant value presented in Table 1. With that, we plot the Reynolds numbers for the flow in two regions: the main part of the cell $\mathrm{Re}_{0}$, and in the end orifice, $\mathrm{Re}^{*}$. At the first point, the velocity is determined by Eq. (8) and the radius $r$ in Eq. (12) is equal to $b=8 \mathrm{~cm}$; at the second point the velocity is determined by Eq. (4) and the radius is equal to $a=0.15 \mathrm{~cm}$. The plot of these two numbers vs the pressure in the cell is presented in Fig. 6.

We note that in all the operational regimes the Reynolds number does not exceed, roughly, 1000, meaning that we never reach regimes of a strong turbulence which would appear if the Reynolds number exceeded a few thousand. This is not to say that the flow will be free of eddies, because we do not take any special measures to make the wall smooth (there will be ports, flanges, etc). The eddies will have the velocity comparable to that of the axial flow. In the main part of the cell these eddies will therefore have velocities much smaller than the sound speed and, accordingly, will not cause any significant change of attenuation. On the other hand, in the exit orifice, the eddies may have velocity which is a fraction of the sound velocity and therefore may cause some density variations. We will show, however, that, because of a small distance occupied by this region, its contribution to the attenuation is negligibly small.

\section{Contribution of the transition region to the attenuation coefficient}

When evaluating the attenuation coefficient $A$ in Sec. 2, we assumed that the gas density is uniform (and equal to $n_{0}$ over the whole volume of the attenuator between the inner surfaces of the beryllium end-plates). However, as Eq. (10) shows, in the vicinity of the end orifices, the density decreases. On the other hand, the density just outside the cell is non-zero, Eq. (11), and contributes to the attenuation. In this section we show that this transition region makes actually a negligible contribution to the total line-density and, therefore, does not affect the attenuation.

Compared to the idealized model, the density on the inner side of the end-plate becomes smaller, thereby reducing the contribution to the line density, whereas to presence of the outer "plume" increases it. Also increasing the line-integral is the gas situated inside the nozzle. The change with respect to the expression $n_{0} L$ to the line density is, therefore, 


$$
\Delta(n L)=2\left\lfloor-\int_{\text {inner }}\left(n_{0}-n\right) d z+0.65 n_{0} h+\int_{\text {outer }} n d z\right\rfloor
$$

The factor "2" accounts for the two ends of the system. The second term is written according to Eq. (5). Using Eqs. (10) and (11) and performing integrations, one finds:

$$
\Delta(n L)=\left(0.86 r_{0}+1.3 h\right) n_{0} \text {. }
$$

For the correction to the attenuation coefficient A one obviously has:

$$
\frac{\Delta A}{A}=\frac{\Delta(n L)}{n L} \ln A=\frac{0.86 r_{0}+1.3 h}{L} \ln A .
$$

Taking $r_{0}=0.15 \mathrm{~cm}, L=600 \mathrm{~cm}$, and $h=0.2 \mathrm{~cm}$ one finds that, even for $A=10^{4}$ (i.e., for $\ln A=9.2), \Delta A / A<0.6 \%$. In other words, the role of the density variation near the exit apertures can be neglected in the evaluation of absorption. The presence of some density fluctuations caused by the compressible turbulence will yield even smaller corrections, because the density fluctuations in the transonic turbulence will hardly exceed $\sim 0.2$ of the average density.

\section{Heating of the gas by the FEL beam}

The FEL beam deposits its energy to the gas mostly via the photoionization process; the initially created photoelectrons have a significant energy (comparable with the energy of x-ray quanta) and cause secondary ionization and excitation of the atoms. A fraction of the energy deposited into the gas is radiated in the line radiation and recombination radiation. Some substantial fraction $\eta(\sim 50 \%)$ is thermalized in the gas. Details of these processes can be found in Ref. [6]. We will discuss here possible effects of this heating on the attenuation coefficient. To get some feel as to how substantial these effects might be, we consider a case of a 2-keV FEL beam, in the regime of the strongest attenuation, $A=10^{4}$. The argon gas density, for a $6 \mathrm{~m}$ long attenuator, will be $n_{0} \approx 5.3 \times 10^{17}$ $\mathrm{cm}^{-3}$ (see Fig. 2).

The beam radius at the location of the gas attenuator will be $r_{b}=0.3 \mathrm{~mm}$ (for the 2-keV beam, Ref. 7). For $A=10^{4}$, half of the beam would be absorbed at a distance $L \ln 2 / \ln A \approx 0.075 L=45 \mathrm{~cm}$. In other words, half of the initial energy $W=2 \mathrm{~mJ}$ will be absorbed in the volume $V=0.13 \mathrm{~cm}^{3}$. The thermal balance equation for a monatomic gas yields the following expression for the temperature increase:

$$
\frac{3}{2} n_{0} k \Delta=\frac{\eta W}{2 V}
$$

where $k$ is the Boltzmann constant. For the aforementioned set of parameters and $\eta=0.5$ one then obtains $\Delta T \approx 330 \mathrm{~K}$. This is a very large temperature increase which would cause an expansion of the heated volume accompanied by a shock wave launched into the surrounding gas. After the radial pressure equilibrium is re-established, one would find a substantial (of order one) density depression at the beam location. This would cause an order one decrease in the overall attenuation factor for the next pulse.

A mitigating factor is the heat conduction in the gas, which would spread the heat over a larger volume before the arrival of the next FEL pulse. For the just discussed set of parameters, the thermal diffusivity in argon is $\chi \sim 10 \mathrm{~cm}^{2} / \mathrm{s}$. Within the time of $\Delta t \sim 10^{-2} \mathrm{~s}$

it will spread the heat over the radius $r \sim \sqrt{2 \chi \Delta t} \sim 3 \mathrm{~mm}$. This would reduce $\Delta T$ at the beam axis by a factor of $\sim 100$, bringing the total attenuation coefficient to within a couple 
of percent from the "dialed in" value. [Note that this density perturbation is localized near the entrance to the gas attenuator at a distance $\sim 45 \mathrm{~cm}$; further downstream, the heating decreases exponentially. ]Another mitigating factor is the presence of eddies (mentioned in Sec. 5), which stir the gas.

We conclude that the heating of the gas by the beam may cause variation of order of a percent or so around the "dialed in" attenuation. A more detailed analysis is required to find firm numbers for various beam energies and gas pressures.

\section{Other sources of uncertainty}

The absorption of X-rays in the gas attenuator is proportional to the particle density. An error of $\Delta n$ in the "dialed in" particle density will give rise to an error of

$$
\frac{\Delta A}{A}=\frac{\Delta n}{n} \ln A
$$

in the relative absorption.

LCLS specifications require an accuracy of $1 \%$ in $\triangle A / A$. According to Eq. (17), to maintain a factor of 2 margin, one has to have $\Delta n / n<0.005 / \ln A$. For the maximum required attenuation, this would mean that the error in the "dialed in" density must be less than $0.05 \%$ (!). This is a difficult task. Perhaps, reaching an uncertainty of $10 \%$ in $\triangle \mathrm{A} / \mathrm{A}$ is more realistic.

A similar consideration shows that the relative temperature variation along the attenuation cell must be kept at the same extremely low level. Indeed, even if we know the density at some specified point exactly, the axial temperature variation will cause the density variation in other points (because of the pressure uniformity). So, we conclude that, in order to provide $1 \%$ accuracy in $\triangle A / A$ at the highest attenuation, one has to maintain the ambient temperature variation along the cell at the level $\Delta T / T<0.0005$ (i.e., less than $0.15 \mathrm{C}$ ).

The gas must be pure, because the presence of an admixture with the different absorption properties would change the attenuation compared to the "dialed in" value. The allowed atomic concentration of admixtures is in the range of $0.05 \%$ (unless they have higher than argon $Z$, in which case the constraint becomes more stringent).

One has also to consider a possible imprecision in the tabulated values [3] of the absorption coefficients for X-rays. According to Ref. [8], a somewhat conservative estimate of the accuracy of the tabulated value of the absorption coefficient is $\sim 4 \%$ at the energies $\sim 800 \mathrm{eV}$ and $\sim 2 \%$ at the energies $\sim 8 \mathrm{keV}$. This would introduce some systematic error (but not irreproducibility) with respect to the "dialed in" attenuation. For the attenuation $A=10^{4}$, the error in $\triangle A$ /A will be $\sim 40 \%$ for the $0.8-\mathrm{keV}$ beam, and $20 \%$ for the 8-keV beam.

\section{Refraction caused by the density non-uniformities}

The density gradient perpendicular to the beam axis causes a refractive deflection of the beam. In this section, we evaluate this effect and show that it is generally small. Let there exist a density gradient in the direction of axis $x$ (produced, e.g., by the 
temperature difference between the bottom and the top of a cell, Sec. 8). We will characterize this gradient by the scale length $\ell$ defined as:

$$
\ell=\left|\frac{1}{n} \frac{\partial n}{\partial x}\right|^{-1}
$$

One can show that the beam deflection angle $\theta$ will be

$$
\Delta \vartheta \approx \frac{f_{1}}{f_{2}} \times \frac{\lambda \ln A}{4 \pi \ell}
$$

where $f_{1}$ and $f_{2}$ are dimensionless parameters characterizing the complex refraction index of the gas [3], $\lambda$ is the wavelength, and $A$ is the attenuation coefficient. [Eq. (19) is derived under the assumption that $f_{1}$ is at least by a factor of 3 greater than $f_{2}$, which is usually the case.]

Consider the maximum attenuation, $A=10^{4}$. For the beam energy of $826 \mathrm{eV}$, one has $\Delta \vartheta \approx 5.4 \times 10^{-7} / \ell(\mathrm{cm})$. For the beam energy of $8260 \mathrm{eV}$, one has $\Delta \vartheta \approx 1.2 \times 10^{-7} / \ell(\mathrm{cm})$. As we do not expect the density variation to exceed $\sim 1 \%$ over the cross-section of the attenuation cell, and assuming that the cell diameter is $\sim 10 \mathrm{~cm}$, we have $\ell>10^{3} \mathrm{~cm}$. Therefore, the refractive deflection does not exceed a fraction of nanoradian and can be ignored.

In a similar way one can show that the effect of thermal fluctuations of the gas density on the beam coherence is negligible.

\section{Mechanical and thermal stresses in the beryllium diaphragm}

The beryllium diaphragm separates the volume where the pressure can reach tens of torrs from the volume where the pressure is by orders of magnitude smaller. This means that the diaphragm will be stressed and somewhat deformed (bulged into the outward direction). The solution of the corresponding elasticity theory problem can be found in Ref. [9]. For a diaphragm of a radius $R$ clamped at the periphery, the maximum displacement occurs near the axis and is equal to

$$
\varsigma=\frac{3 p\left(1-\sigma^{2}\right) R^{4}}{16 E h^{3}}
$$

where $E$ is the Young modulus, and $\sigma$ is the Poisson ratio. [The presence of a small hole, $a<<R$, near the axis does not change the result.] This equation is applicable if the displacement (20) is substantially ( 10 times) less than the slab thickness $h$ (otherwise, a different equation would have to be used). For beryllium, $E=30 \times 10^{10} \mathrm{n} / \mathrm{m}^{2}$, and $\sigma \approx 0.3$. Taking a maximum anticipated pressure of $p=60$ torr $\left(7.7 \times 10^{3} \mathrm{n} / \mathrm{m}^{2}\right)$, we find that the condition $\zeta<0.1 h$ holds if $h / R>0.014$. For $R=1 \mathrm{~cm}$ and $h=0.2 \mathrm{~cm}$, this inequality is satisfied.

The maximum stress is equal to $6 p(R / h)^{2}$ [9]. For our set of parameters, this stress is $1.8 \times 10^{7} \mathrm{n} / \mathrm{m}^{2}$, orders of magnitude less than the yield strength of beryllium, $G=1.5 \times 10^{9} \mathrm{n} / \mathrm{m}^{2}$. So, we conclude that the static pressure will not cause the failure of the exit diaphragms.

The pulsating stresses, caused by turbulent motion of the transonic flow, are small, in the range of $\sim 10 \%$ of the static stresses. Therefore, one should not expect any 
direct damage from turbulent pulsations. Of some concern may be a resonant excitation of the eigenmodes of elastic vibrations of a diaphragm by turbulent flow. Should such an effect be indeed identified during the tests of a prototype, one can mitigate it by adding a damping elements in the zone where the beryllium disc is attached to the external structure. In order to avoid possible concentration of stresses near sharp corners, one should make an aperture with smooth, curved transitions, without sharp angles of the type shown in a rough schematic of Fig.5.

Now we consider a possible thermal damage caused by the heating of the gas during every x-ray pulse, as discussed in Sec. 7. The amount of energy delivered during the pulse $(<2 \mathrm{~mJ})$ is too small to cause any substantial heating of a relatively heavy diaphragm. In addition, beryllium is quite resilient with respect to thermal stresses. So, the direct heating of the diaphragm should not cause any problems. More damaging may be the surface damage caused by the interaction of the beryllium with a hot gas flowing through the nozzle. We comment on that in the following section.

\section{Sputtering and chemical erosion of the diaphragm}

Here we evaluate more accurately the temperature increase in $\mathrm{Ar}$ and $\mathrm{N}_{2}$ in a few centimeter-long zone near the entrance orifice, where the beam would not be attenuated even at $A=10^{4}$.

Table 2. Heating of the gas by the beam pulse with $W=2 \mathrm{~mJ}$

\begin{tabular}{|c|c|c|c|c|c|c|c|c|}
\hline \multirow{2}{*}{$E, \mathrm{keV}$} & \multicolumn{2}{|c|}{0.826} & \multicolumn{2}{c|}{2.0} & \multicolumn{2}{c|}{4.0} & \multicolumn{2}{c|}{8.26} \\
\hline \multirow{2}{*}{$w, \mu \mathrm{m}$} & \multicolumn{2}{|c|}{734} & \multicolumn{2}{c|}{310} & \multicolumn{2}{c|}{206} & \multicolumn{2}{c|}{131} \\
\hline \multirow{2}{*}{$L_{a b s}, \mathrm{~cm}$} & $\mathrm{Ar}$ & $\mathrm{N}_{2}$ & $\mathrm{Ar}$ & $\mathrm{N}_{2}$ & $\mathrm{Ar}$ & $\mathrm{N}_{2}$ & $\mathrm{Ar}$ & $\mathrm{N}_{2}$ \\
\cline { 2 - 10 } & 0.10 & 0.145 & 1.05 & 1.7 & 0.7 & 13.2 & 5.1 & 124 \\
\hline $\begin{array}{c}\text { Q, eV/molecule } \\
(\mathrm{Eq} .(21))\end{array}$ & 0.53 & 0.37 & 0.29 & 0.18 & 0.99 & 0.052 & 0.33 & 0.014 \\
\hline $\begin{array}{c}\Delta T, 10^{30} \mathrm{C} \\
(\mathrm{Eq} .(22), \text { for } \\
\eta=0.5)\end{array}$ & 2.1 & 0.85 & 1.1 & 0.42 & 3.8 & 0.12 & 1.3 & 0.03 \\
\hline
\end{tabular}

Assuming as in Ref. 7 that the radial distribution of the beam intensity is Gaussian,

$$
I=I_{0} \exp \left(-2 r^{2} / w^{2}\right)
$$

where $w$ characterizes the beam width, one can show that the energy absorbed per one atom (for $\mathrm{Ar}$ ) or one molecule (for $\mathrm{N}_{2}$ ) of the gas near the beam axis will be

$$
Q(e V)=\frac{1.48 \times 10^{4} \mathrm{~W}(\mathrm{~mJ})}{L_{a b s}(\mathrm{~cm}) w(\mu \mathrm{m})^{2}}
$$

where $L_{a b s}$ is the e-folding attenuation length at normal conditions (presented in Ref. 3). 
The temperature increase can then be evaluated by the following equations

$$
\Delta T_{A r}\left({ }^{\circ} \mathrm{C}\right)=7.7 \times 10^{3} \eta Q(e V) ; \Delta T_{N_{2}}\left({ }^{\circ} \mathrm{C}\right)=4.64 \times 10^{3} \eta Q(e V)
$$

where $\eta$ is the fraction of the energy that gets thermalized. We have taken into account the fact that argon is a monatomic gas, whereas nitrogen is a diatomic gas. Table 2 contains the results for various x-ray photon energies.

The hottest gas, with the temperature mentioned in Table 2, will be initially present at the beam axis. It can be brought in contact with the surface of the diaphragm by the turbulent eddies. At the lowest densities, where the Knudsen regime is approached, a variety of non-thermal effects may come into play [6].

Very little is known about the interaction of hot, tenuous argon with a cold beryllium surface. The sputtering coefficients for the energies below $1 \mathrm{eV}$ are probably very low, lower than $10^{-6}$. This yields a comfortable margin against the erosion of the beryllium surface. The chemical sputtering in the case of argon is absent.

In the case of nitrogen, chemical reactions on the surface will be boosted by the elevated temperature of the gas. They will lead to a formation of the beryllium nitride, $\mathrm{Be}_{3} \mathrm{~N}_{2}$. A non-uniform deposition may cause the development of high stresses, cracking, and eventual crumbling of the aperture. It is hard to make any definite predictions regarding the rate of the surface degradation. Perhaps, some dedicated experiments could be made with the gas attenuator prototype.

\section{Summary}

A gas attenuator based on the use of argon has attractive features related to the high absorption coefficient in the range up to $8 \mathrm{keV}$. If the differential pumping system can handle the pressure of 60 torr in the attenuation cell, then the attenuator can cover the whole range of the LCLS beam energies, from $0.8 \mathrm{keV}$ to $8 \mathrm{keV}$, with the maximum attenuation of $10^{4}$. An obvious advantage of argon is its low chemical reactivity.

On the down side is the presence of a K-shell jump on the absorption curve which would probably complicate the use of the attenuator in the energy range from 3150 to $3250 \mathrm{eV}$ (see Fig. 7 comparing the attenuation curves for argon and nitrogen). Another disadvantage is the need to introduce, in addition to the nitrogen gas system (which will be used in LCLS anyway), a separate argon gas system.

If the argon attenuator is chosen, one may still want to use some simple solid attenuator in combination with it.

We have not found any problems that would prevent the gas attenuator from a reliable basic operation in the whole range of possible parameters for both argon and nitrogen as a working gas. The mechanical and thermal loads on the beryllium end plates are small. The turbulent fluctuations in the nozzles do not cause any significant change of the attenuation coefficient. A caveat should be made about possible corrosion of the exit apertures under the action of a hot, partly ionized and dissociated nitrogen (Sec. 10). This may become a serious problem for the nitrogen-based attenuator.

Although the basic operation seems to be reliable (with a caveat about corrosion in the case of nitrogen), there may be difficulties with the tuning the attenuators (both argon and nitrogen) to the mode where $1 \%$ accuracy in the attenuation coefficient with respect to the dialed-in value would be achieved. A problem of the errors in $\triangle A / A$ is particularly severe for high attenuations, between $10^{2}$ and $10^{4}$. Reaching such an accuracy 
would require very high accuracy in maintaining the uniformity of the gas along the whole length of the attenuation cell.

Although this report was oriented mainly towards assessing the behavior of argon, the main equations are universal and can be used for any gas (with the appropriate change of the input parameters).

\section{Acknowledgment.}

The authors are grateful to Dr. E. Gullikson (LBL) and Dr. P. Stefan (SLAC) for valuable comments. 


\section{References.}

1. S. Shen. Viewgraphs “LCLS-XTOD Gas Attenuator” presented at the LCLS FAC Meeting (October 27, 2005); UCRL-PRES-21558.

2. D.D. Ryutov, A. Toor. “X-ray attenuation cell.” UCRL-ID-138125, LCLS Technical Note 00-10, May 2000.

3. B.L. Henke, E.M. Gullikson, J.C. Davis. Atomic Data and Nuclear Data Tables, 54, 181 (1993); http://www-cxro.lbl.gov/optical_constants/

4. “A Physicist's Desk Reference,” H.L. Anderson, Ed., AIP, NY, 1989.

5. L.D. Landau, E.M. Lifshitz. "Fluid Mechanics” (NY, Pergamon Press, 1987).

6. D.D. Ryutov, R.M. Bionta, S. Hau-Riege. "Photoluminescence for non-destructive imaging and total energy measurements of X-ray beam in the Linear Coherent Light Source,” LCLS TN, in preparation.

7. R.M. Bionta. Viewgraphs presented at the LCLS Diagnostics and Commissioning Workshop (Sept. 22, 2004).

8. E.M. Gullikson. Private communication to D. Ryutov, December 2005.

9. L.D. Landau and E.M. Lifshitz. “Theory of Elasticity” (NY, Pergamon Press, 1986). 

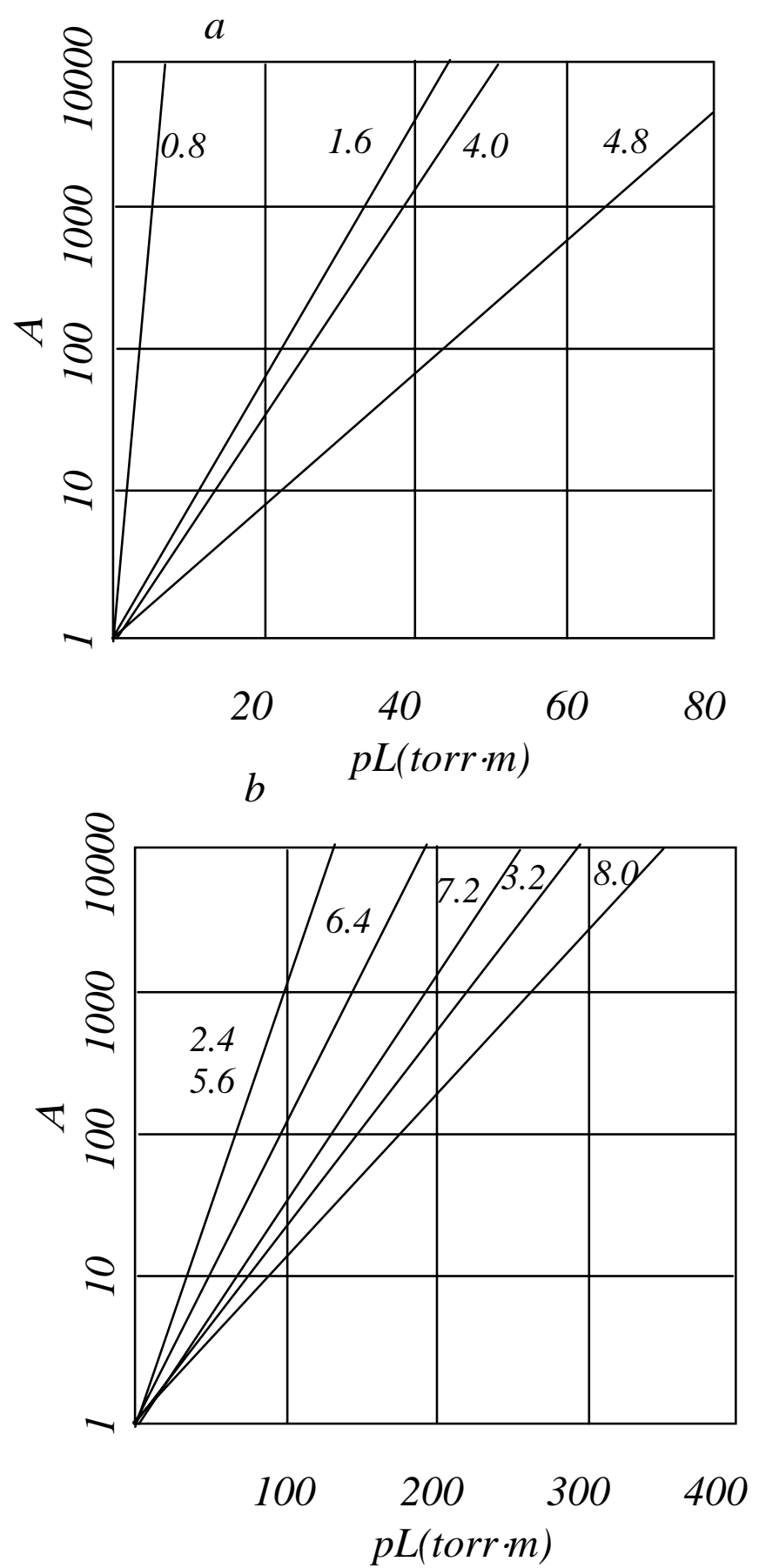

Fig.1 The dependence of the attenuation coefficient $(A)$ on the line-density of argon in the attenuation cell. The numbers by the lines indicate the $\mathrm{X}$-ray energy in $\mathrm{keV}$. We split the figure into two panels because of the difference of the horizontal scales. The lines in panel (b) for the energies 2.4 and $5.6 \mathrm{keV}$ are virtually indistinguishable at this scale. 


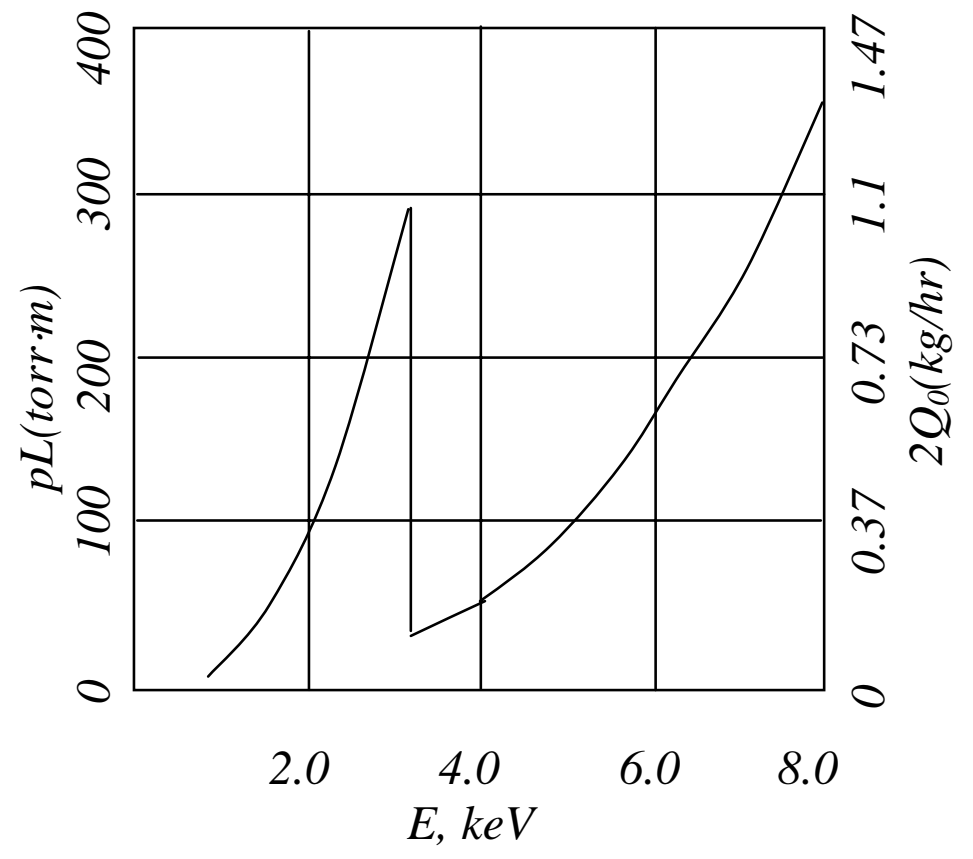

Fig. 2 The argon line density required to produce a $10^{4}$ attenuation at various photon energies (left scale), and the un-inhibited exhaust from two 3-mm diameter orifices from the attenuation cell, for the length of the attenuation cell $L=6 \mathrm{~m}$ (right scale). For the attenuation $A=100$ the vertical scale should be divided by a factor of 2; for the attenuation $A=10$, the vertical scale should be divided by a factor of 4 .

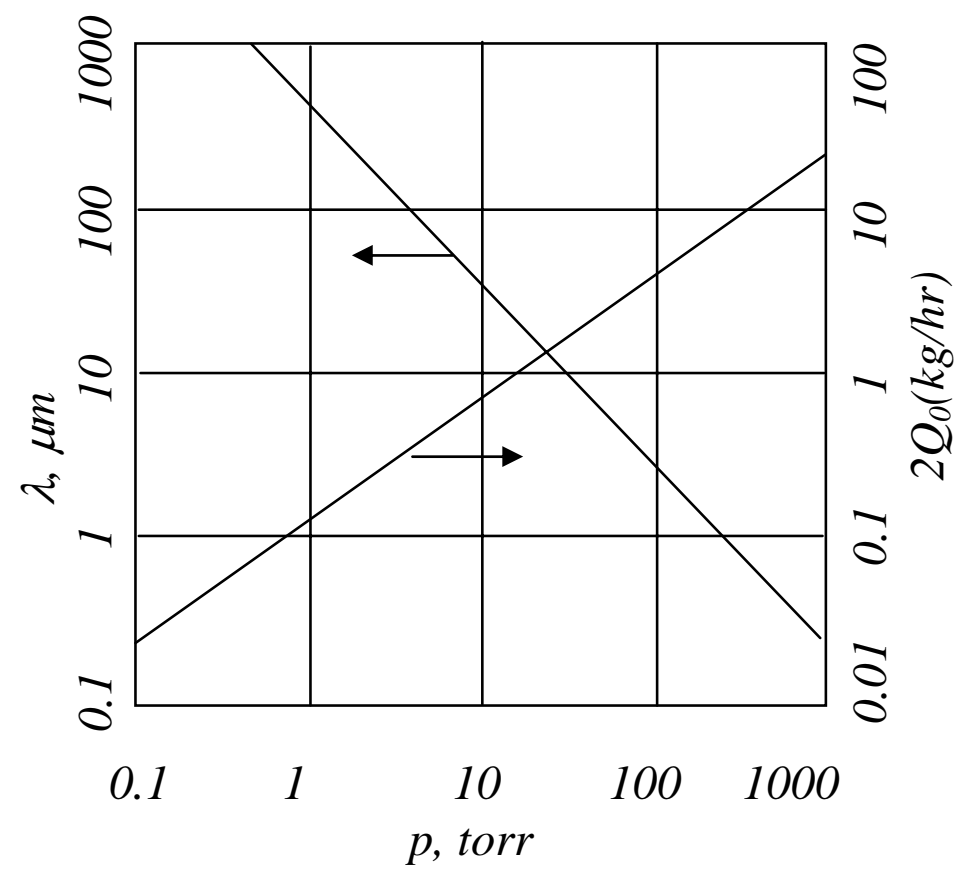

Fig. 3 The mean-free-path (left scale) and the un-inhibited argon outflow from two ends (right scale) vs the argon pressure. 


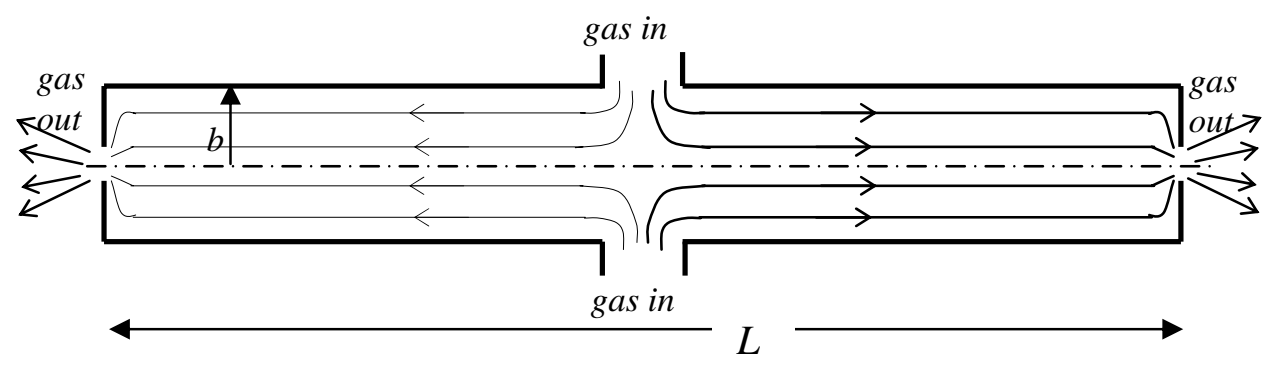

Fig. 4 Schematic of the gas flow in the attenuation cell with two gas inlets situated in the mid-plane (not to scale). The distance $L$ is measured between the inner surfaces of the end plates. The velocity v (Eqs. (8), (9) corresponds to the flow with straight stream-lines between the inlets and end-plates.

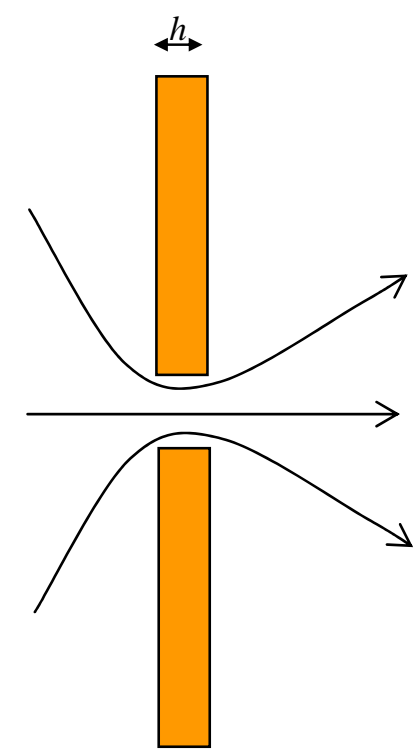

Fig. 5. Streamlines of the average flow near the exit orifice. 


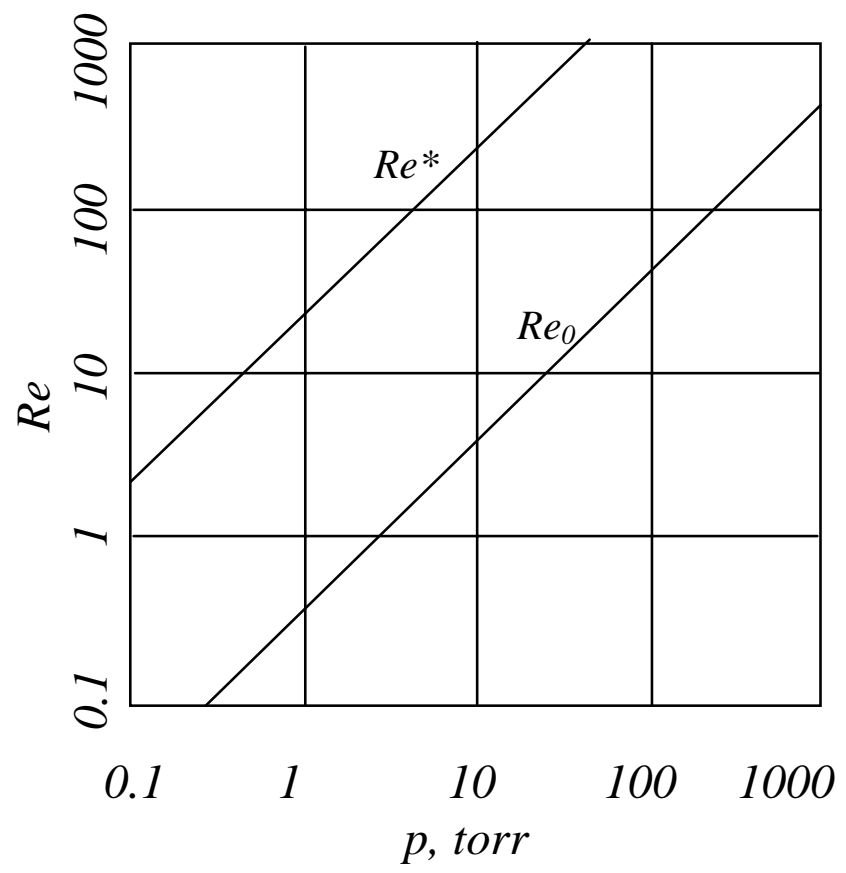

Fig. 6. The Reynolds number for the attenuation cell $\left(\mathrm{Re}_{0}\right)$ and the exit nozzle (Re*). 

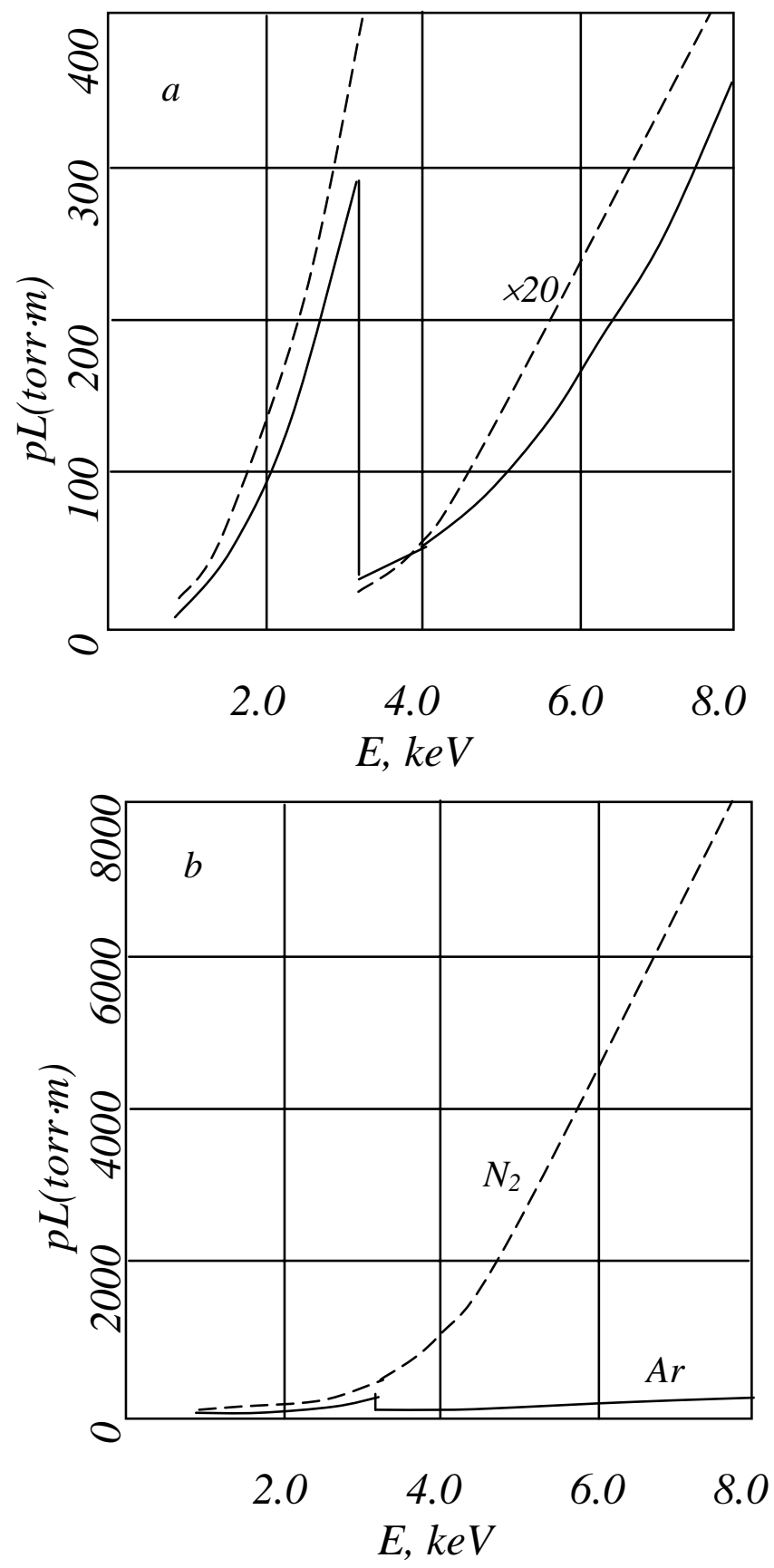

Fig. 7. Comparison of the absorption properties of argon and nitrogen. Shown is the line density required for reaching an attenuation of $10^{4}$ for argon (solid line, the same as in Fig. 2) and for nitrogen (dashed line). In panel $a$, for the energies exceeding $3.2 \mathrm{keV}$, the scale for nitrogen has to be multiplied by 20 (e.g., at $8 \mathrm{keV}$, the line density for nitrogen is $\sim 8500$ torr.m). In panel b a vertical scale identical for both gases is used, to more clearly demonstrate the difference at higher energies. 